(including preoperative biopsy), ultrasound and radiomics features that proved to be different at the univariate analysis on the training set were considered for multivariate analysis and for developing ultrasound-based machine learning assessment models.

Result(s)* For discriminating high risk category versus the other categories one random forest model from the radiomics features (radiomics model), one binary logistic regression model from clinical and ultrasound features (clinical-ultrasound model), and another binary logistic regression model from clinical, ultrasound and previously selected radiomics features (mixed model) were created.

In the validation set, the radiomics model for predicting high risk showed AUC 0.80, sensitivity 58.7\%, specificity $85.7 \%$, positive likelihood ratio $(\mathrm{LR}+) 4.10$ and negative likelihood ratio (LR-) 0.48 ; the clinical-ultrasound model showed AUC 0.87, sensitivity 67.3\%, specificity 89.2\%, LR+ 6.29 and LR- 0.37; and the mixed model showed AUC 0.88, sensitivity $67.3 \%$, specificity $91.0 \%, \mathrm{LR}+7.55$ and LR- 0.36 (table 1).

Conclusion* The mixed model including radiomics, clinical (including preoperative biopsy) and ultrasound features provided the best performance, even if the accuracy was slightly higher in comparison with the model based only on clinical and ultrasound variables. Interestingly, the model based only on radiomics features was able to provide good accuracy to discriminate high risk group versus the others.

\section{MOLECULAR PROFILING OF NSMP HIGH-RISK ENDOMETRIAL CANCERS OF THE PORTEC-3 TRIAL - PROGNOSTIC REFINEMENT AND DRUGGABLE TARGETS}

${ }^{1} \mathrm{~L}$ Vermij ${ }^{*},{ }^{2} \mathrm{M}$ Powell, ${ }^{1} \mathrm{~A}$ Leon-Castillo, ${ }^{3} \mathrm{~S}$ De Boer, ${ }^{4} \mathrm{~L}$ Mileshkin, ${ }^{5} \mathrm{H}$ Mackay, ${ }^{6} \mathrm{~A}$ Leary, ${ }^{7} \mathrm{HW}$ Nijman, ${ }^{8} \mathrm{~N}$ Singh, ${ }^{9} \mathrm{P}$ Pollock, ${ }^{10} \mathrm{P}$ Bessette, ${ }^{11} \mathrm{C}$ Haie-Meder, ${ }^{1} \mathrm{~V}$ Smit, ${ }^{12} \mathrm{R}$ Edmondson,

${ }^{12} \mathrm{E}$ Crosbie, ${ }^{3} \mathrm{R}$ Nout, ${ }^{3} \mathrm{~N}$ Horeweg, ${ }^{3} \mathrm{CL}$ Creutzberg, ${ }^{1 ; 13} \mathrm{~T}$ Bosse. ${ }^{1}$ Leiden University Medical Center (LUMC), Pathology, Leiden, Netherlands; ${ }^{2}$ Barts Health NHS Trust, Clinical Oncology, UK; ${ }^{3}$ Leiden University Medical Center (LUMC), Radiation Oncology, Leiden, Netherlands; ${ }^{4}$ Peter MacCallum Cancer Centre, Medical Oncology, Melbourne, Australia; ${ }^{5}$ Sunnybrook Health Sciences Centre -Odette Cancer Centre, Medical Oncology and Hematology, Toronto, Canada; ${ }^{6}$ Gustave Roussy, Medical Oncology, Villejuif, France; ${ }^{7}$ University Medical Center Groningen, Gynaecology, Groningen, Netherlands; ${ }^{8}$ Barts Health NHS Trust, Pathology, London, UK; ${ }^{9}$ QUT Gardens Point Campus, Institute of Health and Biomedical Innovation, Brisbane City, Australia; ${ }^{10}$ Université de Sherbrooke, Obstetrics and Gynaecology, Sherbrooke, Canada; ${ }^{11}$ Gustave Roussy, Radiation Oncology, Villejuif, France; ${ }^{12}$ St Mary's Hospital, Institute of Cancer Sciences, Manchester, UK; ${ }^{13}$ Leiden University Medical Center, Department of Pathology, Leiden, Netherlands

\subsection{6/ijgc-2021-ESGO.137}

Introduction/Background* The molecular endometrial cancer (EC) classification has proven prognostic value and can direct adjuvant treatment decisions. Despite this, a relatively large group of EC is still molecularly unclassified (NSMP-EC). In this study we aimed to identify biomarkers among high-risk NSMP-EC patients with prognostic and/or predictive relevance. Methodology Paraffin-embedded tumour material $(n=423)$ from the PORTEC-3 HREC trial were available for analysis. All patients with NSMP-EC were selected, hence those without pathogenic POLE mutations, mismatch repair deficiency
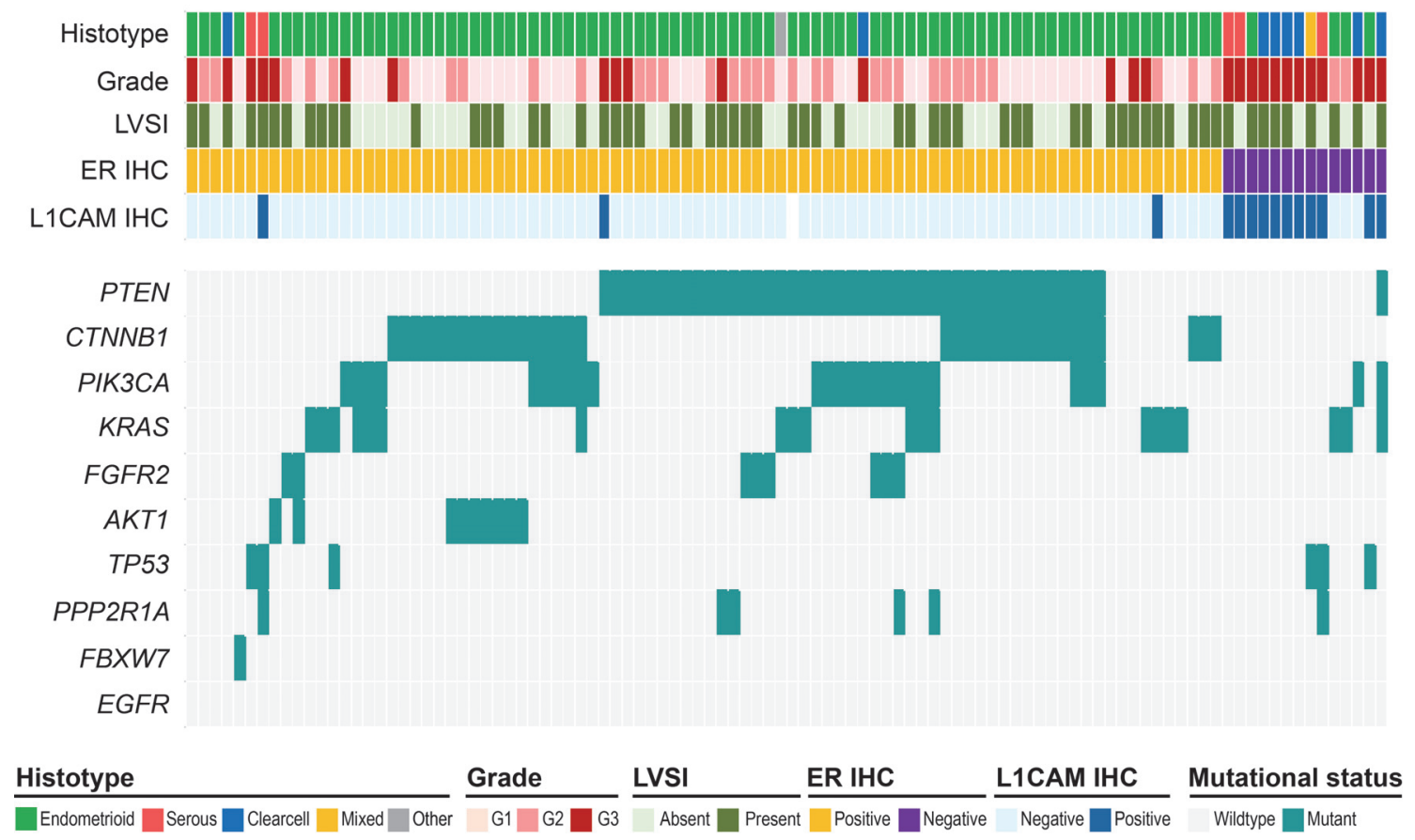

Abstract 397 Figure 1 Overview of clinicopathological and molecular characteristics of NSMP-EC from PORTEC-3 
A

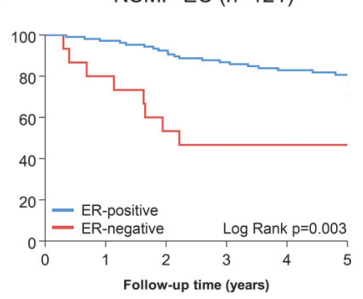

B ER-positive-NSMP EC ( $n=106)$

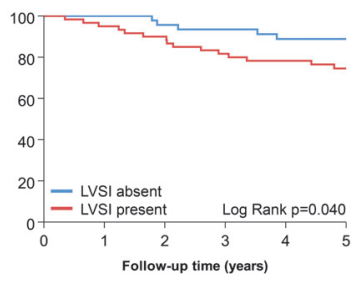

Abstract 397 Figure 2 Time to recurrence within NSMP-EC

and p53-abnormal immunohistochemistry (IHC). Protein expression of L1CAM, ER and PR (ongoing) were analysed by IHC using a $10 \%$ threshold for positivity. Tumour DNA was analysed for pathogenic somatic mutations using a next generation sequencing (NGS) cancer hotspot panel. Time to recurrence was analysed using the Kaplan-Meier method, logrank tests and Cox's proportional hazard models.

Result(s)* In total, 126 NSMP-EC were identified in PORTEC-3, the majority were hormone receptor positive (ER $\mathrm{n}=106 / 121,87.6 \%$, PR will be presented at ESGO2021). L1CAM overexpression was observed in $11.2 \% \quad(n=14 / 125)$ and mutations in CTNNB1-exon-3 were identified in $34.3 \%$ $(n=36 / 105)$. Clustering showed that ER-positive NSMP-EC were predominantly endometrioid EC ( $\mathrm{n}=99$, 93.4\%), low grade $(\mathrm{n}=88,83.0 \%)$ and L1CAM-negative $(\mathrm{n}=102,97.1 \%)$ (figure 1). PIK3CA and KRAS mutations were present in $27.3 \% \quad(n=24)$ and $19.3 \%(n=17)$, respectively. ER-negative NSMP-EC were frequently non-endometrioid ( $\mathrm{n}=11,73.3 \%)$, L1CAM-positive $(\mathrm{n}=11,73.3 \%)$ and rarely harboured PTEN and CTNNB1 mutations $(\mathrm{n}=1,7.1 \%$ and $0 \%$, respectively). ER-positivity was associated with lower risk of recurrence (HR 0.32 [95\%CI 0.14-0.70]; figure 2A), while L1CAM-overexpression and CTNNB1-exon-3 mutations were not (HR 2.25 [95\%CI 0.93-5.43] and HR 1.20 [95\%CI 0.57-2.54], respectively). Multivariable analysis confirmed independent favourable prognostic impact of ER-positivity and LVSI. Figure 2B shows impact of LVSI on time to recurrence among patients with ER-positive NSMP-EC.

Conclusion* The vast majority of NSMP-HREC are ER-positive $(87.6 \%)$ and are likely sensitive to hormonal therapy. Other treatment targets might be found in this subgroup too as $27.3 \%$ had PIK3CA and $19.3 \%$ had KRAS mutations. NSMP-EC with loss of ER-expression were often of non-

endometrioid histology and had a high risk of recurrence. Future studies should investigate whether this subgroup would benefit from other systemic therapies.

\section{PROGNOSTIC RELEVANCE OF THE MOLECULAR ENDOMETRIAL CANCER CLASSIFICATION AMONG PATIENTS STAGED BY LYMPHADENECTOMY AND/OR WITHOUT ADJUVANT TREATMENT}

${ }^{1} \mathrm{~A}$ Leon-Castillo*, ${ }^{2} \mathrm{~N}$ Horeweg, ${ }^{1} \mathrm{E}$ Peters, ${ }^{1} \mathrm{~T}$ Rutten, ${ }^{1} \mathrm{~N}$ Ter Haar, ${ }^{1} \mathrm{~V}$ Smit, ${ }^{3 ; 4} \mathrm{M}$ Boennelycke, ${ }^{3 ; 4} \mathrm{E}$ Høgdall, ${ }^{5} \mathrm{C}$ Høgdall, ${ }^{2} \mathrm{R}$ Nout, ${ }^{2} \mathrm{CL}$ Creutzberg, ${ }^{5} \mathrm{G}$ Ortoft, ${ }^{1 ; 6} \mathrm{~T}$ Bosse. 'Leiden University Medical Center (LUMC), Pathology, Leiden, Netherlands; '2Leiden University Medical Center (LUMC), Radiation oncology, Leiden, Netherlands; ${ }^{3}$ Herlev and Gentofte Hospital, Pathology, Herlev, Denmark; ${ }^{4}$ Copenhagen University Hospital, Rigshospitalet, Pathology, København, Denmark; ${ }^{5}$ Copenhagen University Hospital, Rigshospitalet, Gynecology, København, Denmark; ${ }^{6}$ Leiden University Medical Center, Department of Pathology, Leiden, Netherlands

\subsection{6/ijgc-2021-ESGO.138}

Introduction/Background* The molecular endometrial cancer (EC) classification has proven prognostic impact. However, patients included in previous studies were not always staged by lymphadenectomy (LND) and often received adjuvant treatment (AT). This may have moderated the prognostic effect of the molecular classification. We evaluated the prognostic significance of the molecular classification in high-grade EC patients staged by LND and those without AT.

Methodology Targeted DNA-sequencing for pathogenic POLEexonuclease domain mutations and immunohistochemistry for mismatch repair (MMR) proteins and p53 expression were performed on 412 high-grade EC from the Danish Gynecological Cancer Database 2005-2012 to classify them as POLEultramutated (POLEmut), MMR-deficient (MMRd), p53mutant (p53abn), or no specific molecular profile (NSMP). Patients with stage IV or residual disease after surgery were excluded. Analyses were performed on patients staged by LND and on patients without AT. Time to recurrence analyses were performed using the Kaplan-Meier method, log-rank test and Cox proportional hazard's models. Pre-specified multivariate regression analyses were performed including age, ASA class, stage, lymphovascular space invasion and in the LND subgroup a propensity score to correct for confounding by indication.
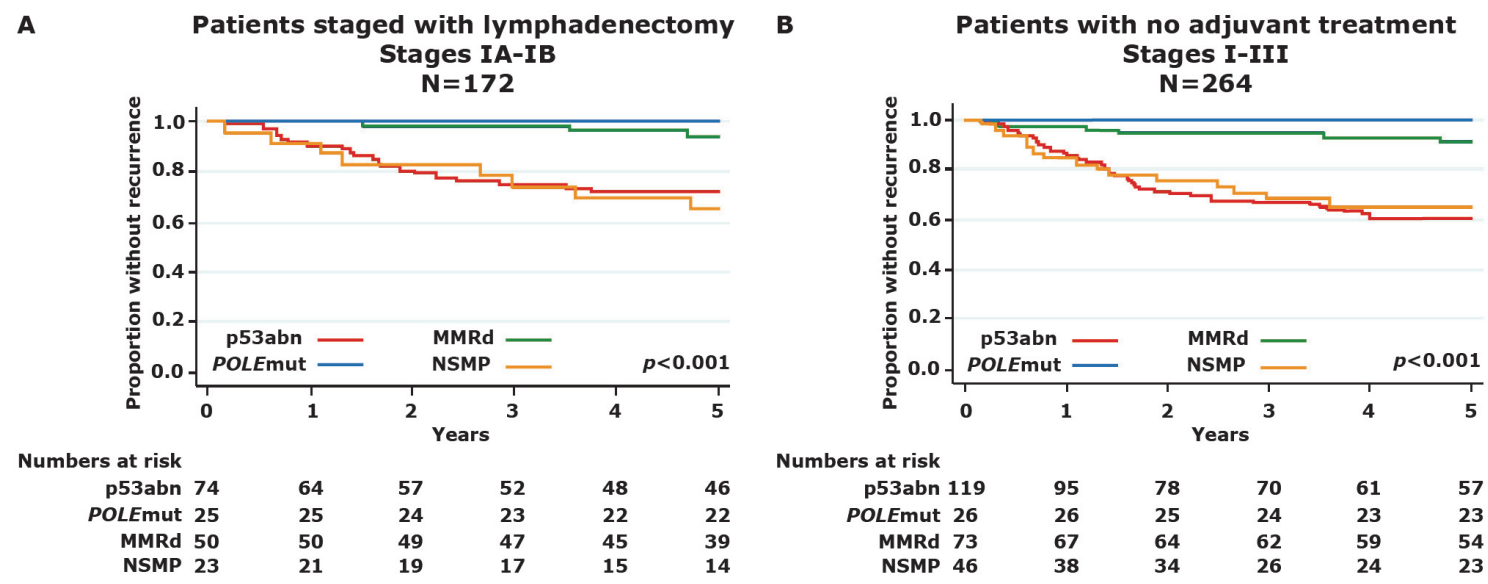

Abstract 406 Figure 1 Time to recurrence of patients staged by lymphadenectomy as stage IA-IB, and of patients with no adjuvant tratment 